\title{
Impact of a Dietary Supplement Containing Rosemary and Daylily on Biochemical Markers of Cognitive Health, Sleep Quality and Related Variables in Men and Women
}

\author{
Richard J. Bloomer¹, John J. MacDonnchadh1, Ryan G. Moran1, Judi Quilici Timmcke², Bolin Qin 2,3 \\ ${ }^{1}$ Cardiorespiratory/Metabolic Laboratory, School of Health Studies, University of Memphis, Memphis, TN, USA \\ ${ }^{2}$ IN-Ingredients, Columbia, TN, USA \\ ${ }^{3}$ Department of Radiation Oncology, University of Maryland School of Medicine, Baltimore, MD, USA \\ Email: rbloomer@memphis.edu
}

How to cite this paper: Bloomer, R.J., MacDonnchadh, J.J., Moran, R.G., Timmcke, J.Q. and Qin, B.L. (2016) Impact of a Dietary Supplement Containing Rosemary and Daylily on Biochemical Markers of Cognitive Health, Sleep Quality and Related Variables in Men and Women. Health, 8, 13071322.

http://dx.doi.org/10.4236/health.2016.813132

Received: August 1, 2016

Accepted: October 9, 2016

Published: October 12, 2016

Copyright $\odot 2016$ by authors and Scientific Research Publishing Inc. This work is licensed under the Creative Commons Attribution International License (CC BY 4.0).

http://creativecommons.org/licenses/by/4.0/

(c) (i) Open Access

\section{Abstract}

Background: We investigated the influence of a botanical agent to improve sleep quality and associated measures in men and women with self-reported difficulty sleeping. Methods: 32 individuals were randomly assigned in double blind manner to ingest a botanical agent (CLOCK ${ }^{\oplus}$, containing Rosemary [Rosmarinus officinalis] and Daylily [Hemerocallis fulva]) or a placebo over a 6-week intervention. During weeks 1 and 2, subjects ingested one serving of the assigned condition, followed by a two-week washout. During weeks 5 and 6, subjects ingested two servings of the condition. The Leeds Sleep Evaluation Questionnaire was used as an outcome measure, as were subjective measures of sleep quality, energy level, and mood. Blood samples collected pre- and post-intervention were assayed for acetylcholine $(\mathrm{ACH})$, brainderived neurotrophic factor (BDNF), irisin, and melatonin. Results: No differences were noted between conditions in measures of sleep ( $p>0.05)$. While no differences of statistical significance were noted in subjective feelings, during weeks 5 and 6 as compared to baseline, subjects assigned to the supplement noted an $8 \%$ increase in attentiveness, an $11 \%$ increase in alertness, a $12 \%$ increase in focus, a $14 \%$ increase in feeling energetic, a $12 \%$ increase in enthusiasm, a $23 \%$ increase in feeling well rested, an $11 \%$ decrease in feeling sluggish, and a $16 \%$ decrease in feeling depressed, without the same improvement observed for subjects in the placebo group. All biochemical measures were increased from pre- to post-treatment with two servings of the supplement; the largest percent increase noted for BDNF (27\%) and the largest effect size noted for irisin $(d=1.36)$. Biochemical values for the placebo condition were unchanged. Conclusions: $\mathrm{CLOCK}^{\circledR}$ may have an impact on certain measurements of 
mood, with a significant impact on the biochemical marker, BDNF. Future studies using a larger sample size and perhaps a cross-over design may help to further clarify the impact of this dietary supplement on aspects of sleep quality, mood, and other related variables.

\section{Keywords}

Rosemary, Daylily, Sleep, Mood, BDNF, irisin

\section{Introduction}

Impaired sleep quality is common among adults with an estimated 50 - 70 million Americans alone claiming poor quality of sleep [1], many of whom use prescription sleep aids [2]. And while prescription drugs are frequently used to aid sleep, aside from melatonin [3], very few dietary supplements with supportive scientific research are currently marketed for this purpose.

A careful review of the literature indicates that certain botanical agents may have promise for improving sleep quality and related health-specific variables. In particular, both Rosemary (Rosmarinus officinalis) and Daylily (Hemerocallis fulva) have been reported to offer benefits. Rosemary is an evergreen branched shrub, naturally occurring primarily around the Mediterranean Sea. The leaves are commonly used as a food additive. The plant has also been traditionally used as an herbal treatment for many conditions [4]. Studies have shown that Rosemary has powerful antioxidant properties [5], and anti-proliferative benefits [6]. Rosemary has been used frequently in traditional medicine as a nonprescription aid for depression [7] [8]. It has been shown that Rosemary has a similar effect to fluoxetine [9], which falls in the SSRI class of antidepressants. More recently, research has shown Rosemary to have an anti-insomnia effect in individuals suffering with opium withdrawal [10]. Further study is needed to examine the possibility of these effects, as well as others, in individuals with non-addiction related sleep difficulties.

The Hemerocallis fulva species of daylily is a perennial plant naturally found in China, Japan, and Korea. Daylily has been used to treat a wide variety of conditions such as jaundice, hemorrhoids, insomnia, and depression. More recently, the plant has been shown to strongly inhibit lipid peroxidation [11], and to have anti-proliferative effects in colon cancer cells [12]. Previous research on animal models has shown Daylily to increase slow-wave sleep during the dark period of the light cycle with no change during the light period [13]. Daylily has been shown to act by increasing the levels of serotonin, dopamine, and norepinephrine in several brain regions [14].

Recently, the dietary supplement called CLOCK ${ }^{\oplus}$, a combination of Rosemary and Daylily, has been evaluated in a small pilot study, with promising outcomes related to the quality of nightly sleep (unpublished findings) and associated changes in mood (e.g. increased vigor, enthusiasm) and cognition (e.g. focus and clarity of thinking). The 
combination of the two ingredients may help to improve sleep quality and associated health benefits (e.g. enhanced mood and relaxation during the waking hours). Interestingly, both Rosemary and Hemerocallis extracts have demonstrated to significantly modulate the expression of circadian clock proteins in C6 animal brain glioma and IPEC-1 cells [15]. Hence, these botanicals may be used as a new approach to improve sleep quality. Moreover, it has been hypothesized that these agents may impact chemicals such as acetylcholine $(\mathrm{ACH})$ and brain-derived neurotrophic factor (BDNF), which may be at least partly responsible for the positive changes observed in mood and cognition (e.g. clarity of thought) following use of the supplements.

In the present study, the combination treatment of Rosemary and Daylily was compared to a placebo condition in men and women with self-reported difficulty sleeping. In this initial trial, we evaluated several variables including sleep quality, mood and associated measures, and biochemical markers of cognitive health.

\section{Methods}

\subsection{Subjects and Screening}

A total of 32 healthy men and women with self-reported difficulty sleeping were enrolled in the study. As this was an initial proof of concept (i.e. pilot) study, there was no power analysis performed. We simply did not have the data for which we could estimate the potential effect size, therefore we included the number of subjects that we believed would be appropriate to generate initial pilot data. Moreover, the sample size of 32 is similar to other study designs in the areas of botanical supplementation. The study data could then be used to help design future studies using this combination of botanical agents. The criteria below were used for study inclusion/exclusion: To be eligible, subjects needed to have a total score of $\geq 8$ on the Insomnia Severity Index [16] and report at least one of following: Routine difficulty falling asleep (cannot get to sleep within 30 minutes); frequent waking ( $\geq 2$ times) during the night; routine experience of having bad dreams; restless sleep (e.g. tossing and turning throughout the night); awakening in the morning feeling tired. It should be noted that our objective was not to enroll those with clinically diagnosed insomnia, but rather, to include those with selfreported difficulty sleeping (with impaired overall sleep quality). We simply used the Insomnia Severity Index as one component of our screening and accepted a relatively low score to allow for subject inclusion. That said, the average score of subjects was close to 16 , indicating that many had poor overall sleep quality, although were not using prescription medication for diagnosed insomnia. Subjects could not have a BMI > $40 \mathrm{~kg} / \mathrm{m}^{2}$, since morbid obesity is known to negatively impact sleep quality [17]. Subjects could not be using dietary supplements designed to improve sleep quality or dietary supplements designed to increase energy. In addition, they could not be using off-the-shelf or prescription medications for purposes of improving sleep quality. They could not be using any other sleep aids. Subjects needed to be non-smokers, and women could not be pregnant or nursing. Subjects could not be consuming alcoholic beverages at a quantity greater than 3 drinks per week. Subjects were recruited by use of re- 
cruitment flyers posted in the Memphis area, in addition to word of mouth recruiting (e.g. snowball sampling), email messages, and presentations to various groups thought to contain potential candidates.

A health history, medication and dietary supplement usage, and physical activity questionnaire was completed by all subjects to determine eligibility. Women were required to take a urine pregnancy test to confirm that they were not pregnant. Prior to participation, each subject was informed of all procedures, potential risks, and benefits associated with the study through verbal and/or written form in accordance with the procedures approved by the University of Memphis Institutional Review Board for Human Subjects Research. Subjects provided written informed consent prior to being admitted to participate.

\subsection{Initial Laboratory Visit: Screening Visit}

During the initial visit to the laboratory, subjects completed the informed consent form, health history and physical activity questionnaires. Subjects' heart rate and blood pressure, height, weight, waist, and hip circumference were measured. Upon completion of the screening, subjects were scheduled for their initial testing visit.

\subsection{Conditions}

Subjects were equally and randomly assigned in double-blind manner to one of two conditions. 1) Placebo (rice bran powder: one capsule (500 mg each) nightly for weeks 1 - 2; two capsules nightly for weeks 5 - 6); or 2) Supplement (one capsule (500 mg each) nightly for weeks 1 - 2; two capsules nightly for weeks 5 - 6). IN-Ingredients, (Columbia, TN, USA) provided the supplement $\left(\mathrm{CLOCK}^{\oplus}\right)$, containing a proprietary blend of Rosemary (Rosmarinus officinalis) and Daylily (Hemerocallis fulva) standardized to ursolic acid. Both supplement and placebo capsules were produced by a contract manufacturer and were of near identical appearance. Capsules were distributed to subjects in unlabeled bottles, with a known quantity of capsules in each bottle. A total of 16 subjects were assigned to each group: 6 men and 10 women were assigned to the placebo, while a total of 6 men and 10 women were assigned to the supplement. Women subjects started the protocol during the initial week of their menstrual cycle in order to control for potential changes in estradiol and other variables across the cycle-as variables related to mood may have been impacted. Although both men and women were enrolled in the study, there were no attempts made to compare the results between genders, as the sample size was simply too small and this was not one of the purposes for this initial work.

Subjects were instructed to ingest the capsule(s) one hour prior to bedtime; ingest the capsule(s) at least 2 hours following their last meal; then turn in their capsule bottle at the end of week 2 . The same bottle was provided back to subjects at the start of week 5 . A two-week washout period was included during weeks 3 and 4, in which subjects did not consume any capsules (placebo or supplement). Knowing the number of capsules contained within each bottle, capsule counting upon bottle return was then used to de- 
termine compliance of intake.

\subsection{Assessment}

Subjects reported to the lab on four different days (over the course of the 6 week period: At baseline and at the end of weeks 2, 4, and 6). The time of day for lab reporting was standardized for each subject across the days of testing and occurred during the early morning hours (i.e., $6 \mathrm{am}-8 \mathrm{am}$ ). Data were collected as indicated below.

1. Heart rate (using an automated unit).

2. Blood pressure (using an automated unit).

3. Respiratory rate (via counting for 60 second period).

4. Blood sample (approximately $7 \mathrm{~mL}$ of blood was collected via venipuncture).

5. Questionnaire related to mood and energy level (using a 10-point scale where $0=$ none and $10=$ extreme). The words provided to subjects were anchored with a 0 and 10. Subjects then indicated the degree to which they "felt" each of these descriptors.

6. Open ended subject comments pertaining to their sleep quality (subjects were asked to describe their overall quality of sleep over the past two weeks, including items such as ease of falling to sleep, frequency of waking at night, tossing and turning during the night, ease of arising in the morning, and energy level upon rising).

In addition to the above laboratory measures, the Leeds Sleep Evaluation Questionnaire [18] was completed on the morning of days 4, 5, 6, and 7 of weeks 2, 4, and 6. Rather than simply have subjects complete the questionnaire in reference to one night, we included four consecutive nights in an attempt to better capture subjects' overall sleep quality and related measures. The average values across days 4 - 7 for each week and for each category of the Leeds Questionnaire was then used in the data analysis. In addition, subjects were asked to rate each morning, the quality of sleep during the prior night and to rate each evening, the quality of the day, in terms of energy level. This was done using a sleep diary and the values were recorded both in the morning and the evening of every day for the entire 6 weeks. Subjects used the following scale to record their ratings: 1 , very bad; 2 , bad; 3 , fair; 4 , good; 5 , very good. The average scores for each set of two weeks were calculated and used in the data analysis.

\subsection{Dietary Records, Physical Activity, and Sleep Conditions}

All subjects were instructed to consume their usual diet throughout the study period and to record all food and drink consumed during the 3 days prior to each test day. Diet records were analyzed for nutrient intake using computer software (Food Processor Pro, Esha Research; Salem, Oregon). Subjects were instructed not to consume alcohol after 7:00 pm or during the 48 hours prior to each test day. Subjects were also instructed not to consume caffeine after 2:00 pm each day. Subjects were instructed to continue with their usual program of physical activity throughout the study period. Finally, subjects were asked to make no changes in their sleep conditions throughout the study period, which would include a change in mattress, pillow, bedding, soundmachine, or other supportive sleep aids. No data were collected related to physical activity 
or sleep conditions.

\subsection{Blood Collection and Biochemical Analysis}

Blood was collected from an antecubital vein. Following collection, samples were processed accordingly and the plasma was stored at $-70^{\circ} \mathrm{C}$ until analyzed. The human enzyme-linked immunosorbent assay (ELISA) kits for $\mathrm{ACH}$ and BDNF were obtained from ABCAM (Cambridge, MA). The ELISA kit for irisin was obtained from Phoenix Pharmaceuticals (Burlingame, CA), while the ELISA kit for melatonin was obtained from antibodies-online.com (Atlanta, GA). Procedures followed the manufactures' guidelines and all samples were assayed in duplicate. Due to our inability to collect blood samples from some subjects for specific reasons (e.g. subjects' fear of needles, difficulty obtaining blood), samples were not available for three subjects in the placebo condition and two subjects in the supplement condition.

\subsection{Statistical Analysis}

The data were analyzed using a condition $\mathrm{x}$ time repeated measures analysis of variance, using all time points of data collection in the overall model. We opted to perform the analysis in this manner, so as to consider any differing effects of a single or double dosage of the assigned condition. Effect sizes were calculated using Cohen's $d$. The data are presented as mean \pm SE. Statistical significance was set at $\mathrm{p} \leq 0.05$.

\section{Results}

All thirty-two subjects successfully completed the protocol. Compliance to capsule intake for subjects assigned to the supplement and placebo was noted to be $96 \%$ and $98 \%$, respectively, with no statistically significant difference noted $(\mathrm{p}>0.05)$. No adverse outcomes were noted with treatment and all subjects tolerated the treatment well, although the following comments were noted from some subjects: one subject reported feelings of weakness, one subject reported abdominal discomfort, one subject reported increased irritability, and eight subjects reported lethargy, with seven of these being in the supplement group.

Subject characteristics are presented in Table 1, with no statistically significant differences noted between conditions $(p>0.05)$. Caffeine intake was higher $(p=0.03)$ for placebo $(244 \pm 63 \mathrm{mg})$ compared to supplement $(102 \pm 16 \mathrm{mg})$. Data for heart rate, blood pressure, and respiratory rate are presented in Table 2. No interaction effects were noted, nor were there any differences between conditions or across time for any measure ( $p>0.05)$. Subjective mood and energy levels were not impacted by treatment across time in a statistically significant way, as can be seen in Table $3(p<0.05)$. However, a condition effect was noted for alertness $(p=0.04)$, with values higher for supplement group compared to placebo. Time effects were noted for the following variables: tiredness $(p=0.001)$, sluggish $(p=0.05)$, energetic $(p=0.02)$, well rested $(p=$ $0.0002)$, and fatigued $(p=0.002)$. In all cases, small improvements were noted across time in the above variables for the supplement group-with beneficial changes noted at 
Table 1. Characteristics of men and women assigned to a supplement (containing Rosemary and Daylily) or a placebo.

\begin{tabular}{lcc}
\hline Variable & Supplement & Placebo \\
\hline Age (yrs) & N = 16 & $\mathrm{~N}=16$ \\
Height $(\mathrm{cm})$ & $172.4 \pm 2.6$ & $26.9 \pm 1.9$ \\
Weight $(\mathrm{kg})$ & $75.2 \pm 4.3$ & $172.3 \pm 3.0$ \\
BMI $\left(\mathrm{kg} \cdot \mathrm{m}^{-2}\right)$ & $25.0 \pm 0.92$ & $73.4 \pm 4.4$ \\
Waist $(\mathrm{cm})$ & $81.3 \pm 2.5$ & $24.5 \pm 1.1$ \\
Hip (cm) & $97.0 \pm 1.9$ & $78.6 \pm 3.5$ \\
Waist:Hip & $0.84 \pm 0.01$ & $94.3 \pm 2.4$ \\
Heart Rate (bpm) & $75.2 \pm 4.5$ & $0.83 \pm 0.02$ \\
Systolic Blood Pressure (mmHg) & $122.6 \pm 2.1$ & $70.3 \pm 2.5$ \\
Diastolic Blood Pressure (mmHg) & $77.4 \pm 2.2$ & $121.9 \pm 2.8$ \\
Years anaerobic exercise training & $4.9 \pm 1.7$ & $76.0 \pm 2.1$ \\
Hours per week anaerobic exercise & $1.7 \pm 0.55$ & $1.4 \pm 0.43$ \\
Years aerobic exercise training & $4.9 \pm 1.3$ & $1.5 \pm 0.42$ \\
Hours per week aerobic exercise & $2.4 \pm 0.45$ & $4.8 \pm 2.2$ \\
Insomnia Index & $15.9 \pm 1.3$ & $2.1 \pm 0.35$ \\
\hline
\end{tabular}

Data are mean \pm SE. No differences of statistical difference were noted for any variable $(p>0.05)$.

Table 2. Heart rate, blood pressure, and respiratory rate of men and women assigned to a supplement (containing Rosemary and Daylily) or a placebo.

\begin{tabular}{lcccc}
\hline Variable & Baseline & Week 2 & Week 4 & Week 6 \\
\hline Heart Rate (bpm) & & & & \\
Supplement & $69.8 \pm 2.8$ & $70.5 \pm 2.9$ & $72.3 \pm 3.3$ & $71.8 \pm 3.1$ \\
Placebo & $73.2 \pm 3.4$ & $75.0 \pm 3.0$ & $72.0 \pm 2.8$ & $69.9 \pm 2.5$ \\
Systolic Blood Pressure (mm Hg) & & & & \\
Supplement & $120.7 \pm 2.8$ & $115.9 \pm 1.7$ & $116.7 \pm 3.5$ & $118.4 \pm 2.2$ \\
Placebo & $121.5 \pm 3.0$ & $123.1 \pm 3.0$ & $119.3 \pm 2.8$ & $120.3 \pm 3.5$ \\
Diastolic Blood Pressure (mm Hg) & & & & \\
Supplement & $75.4 \pm 2.5$ & $73.9 \pm 2.3$ & $76.3 \pm 2.9$ & $72.7 \pm 2.2$ \\
Placebo & $75.7 \pm 2.7$ & $76.1 \pm 2.9$ & $76.9 \pm 2.9$ & $74.6 \pm 3.0$ \\
Respiratory Rate (breaths/min) & & & & \\
Supplement & $17.5 \pm 0.33$ & $17.8 \pm 0.33$ & $17.6 \pm 0.18$ & $17.5 \pm 0.24$ \\
Placebo & $18.4 \pm 0.52$ & $17.9 \pm 0.27$ & $17.9 \pm 0.15$ & $17.8 \pm 0.17$ \\
\hline
\end{tabular}

Values are mean \pm SE. Note: Data collected at Baseline (prior to beginning supplement or placebo) and at the end of weeks 2, 4, and 6 . During weeks 3 and 4, subjects did not ingest any supplement or placebo capsules. No differences of statistical significance were noted for any variable $(\mathrm{p}>0.05)$. 
Table 3. Subjective feelings of men and women assigned to a supplement (containing Rosemary and Daylily) or a placebo.

\begin{tabular}{|c|c|c|c|c|}
\hline Variable & Baseline & Week 2 & Week 4 & Week 6 \\
\hline \multicolumn{5}{|l|}{ Attentive } \\
\hline Supplement & $6.4 \pm 0.39$ & $6.7 \pm 0.41$ & $6.3 \pm 0.39$ & $6.8 \pm 0.34$ \\
\hline Placebo & $6.0 \pm 0.39$ & $6.6 \pm 0.26$ & $6.5 \pm 0.30$ & $5.9 \pm 0.53$ \\
\hline \multicolumn{5}{|l|}{ Tired $^{*}$} \\
\hline Supplement & $6.4 \pm 0.45$ & $5.1 \pm 0.48$ & $5.4 \pm 0.56$ & $5.4 \pm 0.56$ \\
\hline Placebo & $6.4 \pm 0.39$ & $4.4 \pm 0.31$ & $5.1 \pm 0.41$ & $4.1 \pm 0.43$ \\
\hline \multicolumn{5}{|l|}{ Alert $^{* *}$} \\
\hline Supplement & $6.8 \pm 0.36$ & $6.6 \pm 0.33$ & $6.1 \pm 0.49$ & $6.8 \pm 0.31$ \\
\hline Placebo & $5.6 \pm 0.36$ & $6.0 \pm 0.39$ & $6.4 \pm 0.27$ & $6.1 \pm 0.49$ \\
\hline \multicolumn{5}{|l|}{ Groggy } \\
\hline Supplement & $4.8 \pm 0.55$ & $4.1 \pm 0.56$ & $4.2 \pm 0.55$ & $4.1 \pm 0.52$ \\
\hline Placebo & $5.4 \pm 0.54$ & $3.7 \pm 0.44$ & $3.9 \pm 0.57$ & $3.5 \pm 0.61$ \\
\hline \multicolumn{5}{|l|}{ Focused } \\
\hline Supplement & $6.1 \pm 0.36$ & $6.5 \pm 0.42$ & $5.8 \pm 0.53$ & $6.5 \pm 0.47$ \\
\hline Placebo & $5.6 \pm 0.35$ & $6.3 \pm 0.40$ & $6.2 \pm 0.37$ & $6.0 \pm 0.26$ \\
\hline \multicolumn{5}{|l|}{ Sluggish $^{*}$} \\
\hline Supplement & $4.8 \pm 0.57$ & $4.0 \pm 0.51$ & $4.4 \pm 0.57$ & $3.9 \pm 0.55$ \\
\hline Placebo & $5.4 \pm 0.55$ & $3.5 \pm 0.42$ & $3.6 \pm 0.53$ & $3.8 \pm 0.60$ \\
\hline \multicolumn{5}{|l|}{ Energetic $^{*}$} \\
\hline Supplement & $5.4 \pm 0.44$ & $5.6 \pm 0.41$ & $5.6 \pm 0.36$ & $6.4 \pm 0.32$ \\
\hline Placebo & $4.7 \pm 0.34$ & $6.2 \pm 0.43$ & $5.8 \pm 0.35$ & $6.0 \pm 0.41$ \\
\hline \multicolumn{5}{|l|}{ Lethargic } \\
\hline Supplement & $4.1 \pm 0.63$ & $3.9 \pm 0.56$ & $3.3 \pm 0.50$ & $3.4 \pm 0.41$ \\
\hline Placebo & $4.4 \pm 0.50$ & $3.4 \pm 0.46$ & $3.3 \pm 0.50$ & $3.1 \pm 0.54$ \\
\hline \multicolumn{5}{|l|}{ Enthusiastic } \\
\hline Supplement & $6.4 \pm 0.41$ & $6.2 \pm 0.42$ & $5.8 \pm 0.45$ & $6.5 \pm 0.42$ \\
\hline Placebo & $5.8 \pm 0.32$ & $6.8 \pm 0.32$ & $6.5 \pm 0.30$ & $5.9 \pm 0.42$ \\
\hline \multicolumn{5}{|l|}{ Depressed } \\
\hline Supplement & $2.1 \pm 0.45$ & $2.1 \pm 0.5$ & $2.4 \pm 0.57$ & $2.0 \pm 0.47$ \\
\hline Placebo & $2.8 \pm 0.58$ & $2.3 \pm 0.58$ & $2.3 \pm 0.52$ & $2.3 \pm 0.62$ \\
\hline \multicolumn{5}{|l|}{ Well Rested ${ }^{*}$} \\
\hline Supplement & $4.4 \pm 0.40$ & $6.0 \pm 0.52$ & $4.8 \pm 0.32$ & $5.9 \pm 0.50$ \\
\hline Placebo & $3.7 \pm 0.34$ & $5.6 \pm 0.53$ & $5.6 \pm 0.48$ & $6.1 \pm 0.51$ \\
\hline \multicolumn{5}{|l|}{ Fatigued $^{*}$} \\
\hline Supplement & $5.1 \pm 0.47$ & $3.9 \pm 0.55$ & $3.8 \pm 0.45$ & $3.8 \pm 0.59$ \\
\hline Placebo & $5.5 \pm 0.48$ & $3.2 \pm 0.47$ & $4.4 \pm 0.44$ & $3.5 \pm 0.52$ \\
\hline
\end{tabular}

Values are mean \pm SE. Note: Data collected at Baseline (prior to beginning supplement or placebo) and at the end of weeks 2, 4, and 6. During weeks 3 and 4, subjects did not ingest any supplement or placebo capsules. ${ }^{*}$ Condition effect noted for alert $(p=0.04)$. Supplement $>$ Placebo; ${ }^{*}$ Time effects noted for the following variables, which considers the entire group in the analysis: tired $(p=0.001)$, sluggish $(p=0.05)$, energetic $(p=0.02)$, well rested $(p=0.0002)$, and fatigued $(\mathrm{p}=0.002)$; improvements were noted across time in the above variables for the supplement group. No other differences of statistical significance, including interaction effects, were noted for any variable $(\mathrm{p}>0.05)$. 
week 2 and week 6 (Table 3). During weeks 5 and 6 when subjects were assigned double dosage, subjects assigned to the supplement reported an $8 \%$ increase in attentiveness, an $11 \%$ increase in alertness, a $12 \%$ increase in focus, a $14 \%$ increase in a feeling of being energetic, a $12 \%$ increase in enthusiasm, a $23 \%$ increase in feeling well rested, an $11 \%$ decrease in feelings of sluggishness, and a $16 \%$ decrease in feeling depressed-all percent change values relative to week 4 values. These absolute values were not statistically different across time but are presented to provide an overview of the degree of change noted with treatment. Subjects assigned to the placebo experienced small or no positive changes in regards to the above variables. The corresponding effect size for the above variables is as follows: attentiveness $(d=0.34)$, alertness $(d=0.43)$, focus $(d=0.35)$, energetic $(d=0.59)$, enthusiasm $(d=0.40)$, well rested $(d=0.67)$, sluggishness $(d=0.22)$, and depressed $(d=0.19)$.

Data for the Leeds Questionnaire are presented in Table 4. No interaction, time, or condition effects of statistical significance were noted for any measure $(p>0.05)$, with the exception of a time effect noted for "Awake Following Sleep" ( $p=0.007)$. For this variable and considering both conditions, values were higher (improved) at the end of weeks 2 and 6, as compared to week 4. Data related to participants' quality of sleep the prior night and the quality of their day's energy levels are presented in Table 5. There existed no differences of statistical significance for either measure $(\mathrm{p}>0.05)$. Subjects also provided open-ended comments pertaining to their quality of sleep over the prior two weeks and these were similar for both conditions.

Table 4. Leeds Sleep Evaluation Questionnaire data of men and women assigned to supplement (containing Rosemary and Daylily) or a placebo.

\begin{tabular}{cccc}
\hline Variable & Week 2 & Week 4 & Week 6 \\
\hline $\begin{array}{c}\text { Getting to Sleep } \\
\text { Supplement }\end{array}$ & $173.3 \pm 11.4$ & $144.0 \pm 8.0$ & $173.7 \pm 12.6$ \\
Placebo & $164.9 \pm 6.9$ & $150.4 \pm 7.7$ & $179.3 \pm 12.3$ \\
Quality of Sleep & & & \\
Supplement & $118.8 \pm 9.2$ & $93.7 \pm 6.4$ & $112.4 \pm 10.2$ \\
$\quad$ Placebo & $115.8 \pm 7.3$ & $100.0 \pm 8.3$ & $123.0 \pm 9.8$ \\
Awake Following Sleep & & & $97.1 \pm 8.9$ \\
Supplement & & $94.3 \pm 4.2$ & $113.2 \pm 8.9$ \\
Placebo & $96.7 \pm 10.4$ & $96.5 \pm 7.5$ & \\
Behavior Following Waking & $109.0 \pm 6.8$ & & $144.6 \pm 12.3$ \\
Supplement & & $143.7 \pm 8.8$ & $159.1 \pm 11.9$ \\
Placebo & $156.0 \pm 14.1$ & $145.9 \pm 8.1$ & \\
\hline
\end{tabular}

Values are mean \pm SE. Note: Data collected during days $4,5,6$, and 7 of weeks 2,4 , and 6 . The average values for each 4 day period were used in data analysis and are presented above. During weeks 3 and 4 , subjects did not ingest any supplement or placebo capsules. ${ }^{*}$ Time effect for "Awake Following Sleep" $(\mathrm{p}=0.007)$, which considers the entire group in the analysis; Values higher at the end of weeks 2 and 6 as compared to week 4 . No other differences of statistical significance were noted for any variable $(\mathrm{p}>0.05)$. 
Table 5. Quality of sleep during the prior night and quality of the day in terms of energy level of men and women assigned to a supplement (containing Rosemary and Daylily) or a placebo.

\begin{tabular}{cccc}
\hline Variable & Week 2 & Week 4 & Week 6 \\
\hline Quality of Sleep & & & \\
Supplement & $3.5 \pm 0.14$ & $3.4 \pm 0.11$ & $3.7 \pm 0.17$ \\
Placebo & $3.3 \pm 0.16$ & $3.2 \pm 0.12$ & \\
Quality of Day & & & $3.7 \pm 0.14$ \\
Supplement & $3.6 \pm 0.13$ & $3.5 \pm 0.10$ & $3.7 \pm 0.09$ \\
Placebo & $3.5 \pm 0.13$ & $3.5 \pm 0.14$ & \\
\hline
\end{tabular}

Values are mean \pm SE. Note: Data were collected every day during the entire 6 week period. The average values for each week were used in the data analysis and are presented above. During weeks 3 and 4 , subjects did not ingest any supplement or placebo capsules. No differences of statistical significance were noted for either variable $(p>0.05)$.

With regards to biochemical markers, no condition by time interactions were noted ( $p>0.05$ ) and data are shown in Table 6. Subjects taking two capsules per day demonstrated significantly higher BDNF, irisin, and melatonin levels from pre to post treatment, week 4 to week $6(\mathrm{p}<0.05)$. The corresponding effect size for the above variables is as follows: acetylcholine $(d=0.40), \operatorname{BDNF}(d=0.44)$, irisin $(d=1.36)$, and melatonin $(d=0.84)$.

Dietary data were not different between conditions or across time $(\mathrm{p}>0.05)$, with exception of a condition effect for calcium $(\mathrm{p}=0.04)$ and magnesium $(\mathrm{p}=0.04)$, both of which were higher for placebo as compared to supplement. Dietary data are not shown.

\section{Discussion}

To our knowledge, the present study was the first controlled evaluation of Rosemary (Rosmarinus officinalis) and Daylily (Hemerocallis fulva) in relation to sleep quality and associated variables in men and women with self-reported difficulty sleeping. Although no findings of statistical significant were noted for sleep quality and no condition $\mathrm{x}$ time interaction effects were noted for any outcome measure, certain subjective measures appeared to be improved for subjects assigned to the supplement condition-in particular while ingesting two capsules per day. While some positive changes were noted in these variables for subjects assigned to the placebo condition, in some cases no improvements were noted, and in others, values actually were worse following placebo treatment as compared to before. Indeed, subjective measures vary considerably from one subject to the next, with some noting major improvements and other noting little to no change in some measures. The clinical significance and practical relevance of these improvements is presently unknown but deserves attention in future work involving a larger subject sample and perhaps a longer time course of treatment.

Aside from the above, both subjective numeric data and the open ended comments provided by subjects were similar between supplement and placebo groups. When 
Table 6. Biochemical markers of men and women assigned to a supplement (containing Rosemary and Daylily) or a placebo.

\begin{tabular}{ccccc}
\hline Variable & Baseline & Week 2 & Week 4 & Week 6 \\
\hline Acetylcholine $($ nmoll $L)$ & & & & \\
Placebo & $2.45 \pm 0.20$ & $2.44 \pm 0.21$ & $2.39 \pm 0.18$ & $2.43 \pm 0.19$ \\
Supplement & $2.20 \pm 0.15$ & $2.42 \pm 0.12$ & $2.29 \pm 0.06$ & $2.49 \pm 0.18$ \\
BDNF(ng/mL) & & & & \\
Placebo & $36.5 \pm 4.2$ & $37.3 \pm 5.0$ & $37.6 \pm 3.4$ & $38.0 \pm 3.7$ \\
Supplement & $34.7 \pm 4.0$ & $36.4 \pm 4.9$ & $32.1 \pm 3.4$ & $41.1 \pm 2.8 * *$ \\
irisin $($ ng/mL $)$ & & & & \\
Placebo & $29.9 \pm 0.6$ & $30.0 \pm 0.9$ & $29.6 \pm 1.2$ & $29.7 \pm 1.1$ \\
Supplement & $29.6 \pm 0.6$ & $30.1 \pm 0.7$ & $29.0 \pm 0.4$ & $30.8 \pm 0.5^{* *}$ \\
Melatonin $(p g / m L)$ & $1029.1 \pm 13.1$ & $1033.5 \pm 5.7$ & $1036.2 \pm 8.5$ & $1032.2 \pm 15.9$ \\
Placebo & $1051.7 \pm 7.0$ & $1049.5 \pm 3.7$ & $1036.5 \pm 8.9$ & $1057.3 \pm 2.4^{* *}$ \\
Supplement & & & & \\
\hline
\end{tabular}

Values are mean $\pm S E ; n=13$ for placebo group and $n=14$ for supplement group. ${ }^{* *}$ With two capsules of the supplement, BDNF, irisin, and melatonin levels were all higher from pre- to post-treatment $(p<0.05)$. No other differences of statistical significance were noted for any variable $(p>0.05)$.

viewing the supplement results exclusively, it was apparent for many variables that improvements were noted. For example, both "getting to sleep" and "quality of sleep" values for the Leeds questionnaire improved by $20 \%$ with the supplement condition (from week 4 to week 6, while using two capsules per day; Table 4). However, similar percent improvements were noted for the placebo condition. Likewise, open ended comments for subjects in the supplement condition were positive with regards to their sleep quality (comments not shown). However, in a similar manner as for the Leeds questionnaire, comments were also positive for the placebo group. Interestingly, one subject assigned to the placebo condition stated that two capsules per day provided too strong of an effect. We are uncertain as to what, if any relationship exists between these comments and the biochemical data, as subjective comments were similar for subjects in both conditions. More careful analysis done in future work, inclusive of a larger sample of subjects is needed to more clearly delineate the association between biochemical measures and subjective comments provided by subjects.

Considering the above, it was apparent viewing the data that a placebo effect may have existed for some outcomes. The placebo effect is well-described [19]-[21] and continues to pose problems for investigators who choose to use a placebo controlled design with attempts to find statistical significance between active and placebo conditions. In an investigation focused on the effectiveness of another dietary supple- ment proposed to impact sleep (Humulus lupulus), variables were noted to improve similarly with both the placebo and supplement condition [22], much like in the present design. Another study compared dextromethorphan, diphenhydramine, and a placebo evaluated sleep 
quality in children. It was found that all three groups demonstrated significant improvements without any differences noted between them [23]. Rosemary has previously been shown to act in a similar biological manner to fluoxetine when ingested. Fluoxetine itself was previously examined, comparing it to a placebo and the results showed that the placebo exhibited similar neurological changes to fluoxetine in nine of a possible thirteen brain regions [24]. Clearly, this issue of a placebo effect may, if present in the present study, have impaired our ability to statistically note differences between conditions for sleep-related measures.

Aside from the sleep-related outcomes, the supplement promoted a small $(\sim 3 \%)$ reduction in blood pressure (Table 2). This may be linked to the sedative effects of the supplement, as subjective comments also included a $16 \%$ decrease in feeling depressed. Longer-term treatment with the supplement may be warranted for purposes of determining the potential impact of this agent on both blood pressure regulation and alleviating symptoms of depression.

Related to our subjective mood data, biochemical variables were noted to be impacted by the supplement (Table 6). Most notably, BDNF was increased by appro- ximately $27 \%$ with treatment of two capsules per day of the supplement. Interestingly, this percent increase is similar to what has been noted for acute strenuous exercise [25] and we are unaware of other dietary supplements that have been shown to result in a measurable increase in BDNF. Brain-derived neurotrophic factor, a member of neurotrophin family, exists in the brain, as well as in other tissue such as skeletal muscle. It plays a central role in mood disorders [26] and is associated with improved cognitive performance [27] and alertness [28]. The significant increase in BDNF with treatment of the supplement may provide mechanistic evidence for the improvement in our subjective measures of mood, such as alertness, focus, and enthusiasm.

In addition to the increase in BDNF, we noted an increase in plasma irisin, a novel myokine, which has been involved in the pathogenesis of type 2 diabetes, metabolic syndrome, and metabolic health in general. Acute exercise appears to elevate irisin and the response may be mode dependent [29]. While the present study did not seek to measure outcomes related to metabolic health and associated irisin levels, this may be the focus of future work involving the combination treatment of Rosemary and Daylily. Although the overall increase in Irsin did not appear robust, the effect size was quite large $(d=1.36)$ and this measure may be worth investigating in future studies. Finally, both $\mathrm{ACH}$ and melatonin levels were elevated slightly with supplementation; however, only melatonin was increased in a statistically significant manner $(\mathrm{p}<0.05)$, while a trend was noted for ACH $(\mathrm{p}=0.058)$. We are uncertain of the direct impact of these changes on health outcomes of our subjects. However, increases in these measures may help to explain our findings for improved mood and associated variables. Finally, as other neurotransmitters may be influenced by the botanical agent, future studies may seek to include variables aside from those included in the present study (e.g. GABA, 5-hydroxytryptophan).

We need to acknowledge some limitations of this work related to the sleep assess- 
ment. First, subjects did not record sleep duration or naptime throughout the course of the study. Second, while we believe that use of the Leeds Questionnaire coupled with the sleep diary was appropriate, future studies may consider using a more detailed sleep diary, documenting the onset of sleep, number of awakenings, number of hours slept, and naps taken throughout the day. Specifically, future work might use the Consensus Sleep Diary, which may be viewed as the gold standard for insomnia research. Our failure to do so in the present study may be considered a limitation of this work.

\section{Conclusion}

CLOCK $^{\oplus}$, a novel plant-based dietary supplement, may improve some measures of mood, perceived energy, and associated variables in men and women with self-reported difficulty sleeping. The supplement also results in an increase in the biochemical measures $\mathrm{ACH}, \mathrm{BDNF}$, irisin, and melatonin. Related to the biochemical findings, this is the first study that we are aware of to report such a significant increase in BDNF with treatment, as well as an increase in irisin. The interplay between sleep quality and quantity, mood and perceived energy level, and cognitive function has been previously described [30]-[32]. Additional work focused on the mechanisms of effect of the botanical agent to improve measures of sleep quality, mood, and cognitive health may be warranted. These effects may warrant further investigation, possibly extending the time frame of treatment and including a cross-over design, in an attempt to decrease subject variability by having each individual serve as their own control. Finally, due to the potential influence of dietary intake on sleep quality and related variables, greater care may be taken to control dietary intake, in particular caffeine intake.

\section{Authors' Contributions}

RJB was responsible for the study design, statistical analyses, and manuscript preparation. JJM and RGM were responsible for subject recruitment and retention, data collection, data entry, and assistance with manuscript preparation. JQT was responsible for assistance with the study design and manuscript editing. BQ was responsible for performing the biochemical analyses and assistance with statistical analyses and manuscript editing. All authors read and approved of the final manuscript.

\section{Conflict of Interest}

Financial support for this work was provided in part by IN-Ingredients. JQT and BQ are employees of IN-Ingredients. None of the other authors have a financial interest in this company. RJB has received research funding or acted as consultant to other nutraceutical and dietary supplement companies. All other authors declare no competing interests.

\section{Funding}

Financial support for this work was provided in part by IN-Ingredients. 


\section{Acknowledgements}

Funding for this work was provided in part by IN-Ingredients (Columbia, TN) and the University of Memphis.

\section{References}

[1] Institute of Medicine (2006) Sleep Disorders and Sleep Deprivation: An Unmet Public Health Problem. The National Academies Press, Washington DC.

[2] Chong, Y., Fryer, C.D. and Gu, Q. (2013) Prescription Sleep Aid Use among Adults: United States, 2005-2010. NCHS Data Brief, 127, 1-8.

[3] Turek, F.W. and Gillette, M.U. (2004) Melatonin, Sleep, and Circadian Rhythms: Rationale for Development of Specific Melatonin Agonists. Sleep Medicine, 5, 523-532. http://dx.doi.org/10.1016/j.sleep.2004.07.009

[4] Al-Sereitia, M., Abu-Amerb, K. and Sena, P. (1999) Pharmacology of Rosemary (Rosmarinus officinialis Linn.) and Its Therapeutic Potentials. Indian Journal of Experimental Biology, 37, 124-131.

[5] Erkan, N., Ayranci, G. and Ayranci, E. (2008) Antioxidant Activities of Rosemary (Rosmarinus officinalis L.) Extract, Blackseed (Nigella sativa L.) Essential Oil, Carnosic Acid, Rosmarinic Acid and Sesamol. Food Chemistry, 110, 76-82.

http://dx.doi.org/10.1016/j.foodchem.2008.01.058

[6] Tai, J., Cheung, S., Wu, M. and Hasman, D. (2012) Antiproliferation Effect of Rosemary (Rosmarinus officinalis) on Human Ovarian Cancer Cells in Vitro. Phytomed, 19, 436-443. http://dx.doi.org/10.1016/j.phymed.2011.12.012

[7] Foster, S. and Johnson, R.L. (2006) Rosemary. In: National Geographic Desk Reference to Natural Medicine. National Geographic Society Publisher, Washington DC, 316.

[8] Machado, D.G., Cunha, M.P., Neis, V.B, Balen, G.O., Colla, A.R. and Grando, J. (2012) Rosmarinus officinalis L. Hydroalcoholic Extract, Similar to Fluoxetine, Reverses De- pressive-Like Behavior without Altering Learning Deficit in Olfactory Bulbectomized Mice. Journal of Ethnopharmacology, 143, 158-169. http://dx.doi.org/10.1016/j.jep.2012.06.017

[9] Machado, D.G., Bettio, L.E., Cunha, M.P., Capra, J.C., Dalmarco, J.B. and Pizzolatti, M.G. (2009) Antidepressant-Like Effect of the Extract of Rosmarinus officinalis in Mice: Involvement of the Monoaminergic System. Progress in Neuro-Psychopharmacology \& Biological Psychiatry, 33, 642-650. http://dx.doi.org/10.1016/j.pnpbp.2009.03.004

[10] Solhi, H., Salehi, B., Alimoradian, A., Pazouki, S., Taghizadeh, M. and Saleh, A.M. (2013) Beneficial Effects of Rosmarinus officinalis for Treatment of Opium Withdrawal Syndrome during Addiction Treatment Programs: A Clinical Trial. Addict Health, 5, 90.

[11] Zhang, Y., Cichewicz, R.H. and Nair, M.G. (2004) Lipid Peroxidation Inhibitory Compounds from Daylily (Hemerocallis fulva) Leaves. Life Science, 75, 753-763. http://dx.doi.org/10.1016/j.lfs.2004.03.002

[12] Cichewicz, R.H., Zhang, Y., Seeram, N.P. and Nair, M.G. (2004) Inhibition of Human Tumor Cell Proliferation by Novel Anthraquinones from Daylilies. Life Science, 74, 17911799. http://dx.doi.org/10.1016/j.lfs.2003.08.034

[13] Uezu, E. (1998) Effects of Hemerocallis on Sleep in Mice. Psychiatry and Clinical Neurosciences, 52, 136-137. http://dx.doi.org/10.1111/j.1440-1819.1998.tb00992.x

[14] Lin, S.H., Chang, H.C., Chen, P.J., Hsieh, C.L., Su, K.P. and Sheen, L.Y. (2013) The Antidepressant-Like Effect of Ethanol Extract of Daylily Flowers in Rats. Journal of Alternative and Complementary Medicine, 3, 53. http://dx.doi.org/10.4103/2225-4110.106548 
[15] Qin, B., Shea-Donohue, T., Liu, C., Quilici Timmcke, J. and Anderson, R.A. (2016) The Regulation in Key Protein Expression Related to Circadian Rhythms with a Natural Compound Containing Rosemary and Hemerocallis fulva Extract in Vitro. FASEB Journal, 30, No. 1. http://dx.doi.org/10.1096/fj.1530-6860

[16] Morin, C.M, Belleville, G., Bélanger, L. and Ivers, H. (2011) The Insomnia Severity Index: Psychometric Indicators to Detect Insomnia Cases and Evaluate Treatment Response. Sleep, 34, 601-608.

[17] Tishler, P.V., Larkin, E.K., Schluchter, M.D. and Redline, S. (2003) Incidence of Sleep- Disordered Breathing in an Urban Adult Population: The Relative Importance of Risk Factors in the Development of Sleep-Disordered Breathing. JAMA, 289, 2230-2237. http://dx.doi.org/10.1001/jama.289.17.2230

[18] Parrott, A.C. and Hindmarch, I. (1980) The Leeds Sleep Evaluation Questionnaire in Psychopharmacological Investigations: A Review. Psychopharmacology, 71, 173-179.

http://dx.doi.org/10.1007/BF00434408

[19] Donnelly, G.F. (2004) The Placebo Effect and Holistic Interventions. Holistic Nursing Practice, 18, 238-241. http://dx.doi.org/10.1097/00004650-200409000-00006

[20] Eccles, R. (2007) The Power of the Placebo. Current Allergy and Asthma Reports, 7, 100104. http://dx.doi.org/10.1007/s11882-007-0006-2

[21] Finniss, D.G., Kaptchuk, T.J., Miller, F. and Benedetti, F. (2010) Biological, Clinical, and Ethical Advances of Placebo Effects. Lancet, 375, 686-695.

http://dx.doi.org/10.1016/S0140-6736(09)61706-2

[22] Cornu, C., Remontet, L., Noel-Baron, F., Nicolas, A., Feugier-Favier, N., Roy, P. and Claustrat, B. (2010) A Dietary Supplement to Improve the Quality of Sleep: A Randomized Placebo Controlled Trial. BMC Complementary and Alternative Medicine, 10, 29. http://dx.doi.org/10.1186/1472-6882-10-29

[23] Paul, I.M., Yoder, K.E., Crowell, K.R., Shaffer, M.L., McMillan, H.S. and Carlson, L.C. (2004) Effect of Dextromethorphan, Diphenhydramine, and Placebo on Nocturnal Cough and Sleep Quality for Coughing Children and Their Parents. Pediatrics, 114, e85-e90. http://dx.doi.org/10.1542/peds.114.1.e85

[24] Mayberg, H.S., Silva, J.A., Brannan, S.K., Tekell, J.L., Mahurin, R.K. and McGinnis, S. (2002) The Functional Neuroanatomy of the Placebo Effect. American Journal of Psychiatry, 159, 728-737. http://dx.doi.org/10.1176/appi.ajp.159.5.728

[25] Ferris, L.T., Williams, J.S. and Shen, C.L. (2007) The Effect of Acute Exercise on Serum Brain-Derived Neurotrophic Factor Levels and Cognitive Function. Medicine \& Science in Sports \& Exercise, 39, 728-734. http://dx.doi.org/10.1249/mss.0b013e31802f04c7

[26] Hashimoto, K., Shimizu, E. and Iyo, M. (2004) Critical Role of Brain-Derived Neurotrophic Factor in Mood Disorders. Brain Research Reviews, 45, 104-114. http://dx.doi.org/10.1016/j.brainresrev.2004.02.003

[27] Komulainen, P., Pedersen, M., Hänninen, T., Bruunsgaard, H., Lakka, T.A. and Kivipelto, M. (2008) BDNF Is a Novel Marker of Cognitive Function in Ageing Women: The DR's EXTRA Study. Neurobiology of Learning and Memory, 90, 596-603. http://dx.doi.org/10.1016/j.nlm.2008.07.014

[28] Mikoteit, T., Hemmeter, U., Eckert, A., Brand, S., Bischof, R. and Delini-Stula, A. (2015) Improved Alertness Is Associated with Early Increase in Serum Brain-Derived Neurotrophic Factor and Antidepressant Treatment Outcome in Major Depression. Neuro- psychobiology, 72, 16-28. http://dx.doi.org/10.1159/000437439

[29] Tsuchiya, Y., Ando, D., Takamatsu, K. and Goto, K. (2015) Resistance Exercise Induces a 
Greater Iris in Response than Endurance Exercise. Metabolism, 64, 1042-1050.

http://dx.doi.org/10.1016/j.metabol.2015.05.010

[30] McClung, C.A. (2013) How Might Circadian Rhythms Control Mood? Let Me Count the Ways. Biological Psychiatry, 74, 242-249. http://dx.doi.org/10.1016/j.biopsych.2013.02.019

[31] Wirz-Justice, A. (2006) Biological Rhythm Disturbances in Mood Disorders. International Clinical Psychopharmacology, 21, S11-S15.

http://dx.doi.org/10.1097/01.yic.0000195660.37267.cf

[32] Kohyama, J. and Frank, M.G. (2016) Neural Basis of Brain Dysfunction Produced by Early Sleep Problems. Brain Sciences, 6, 5. http://dx.doi.org/10.3390/brainsci6010005

\section{Abbreviations and Acronyms}

ACH: Acetylcholine

BDNF: Brain-derived neurotrophic factor

ELISA: Enzyme-linked immunosorbent assay

GABA: Gamma amino butyric acid

Submit or recommend next manuscript to SCIRP and we will provide best service for you:

Accepting pre-submission inquiries through Email, Facebook, LinkedIn, Twitter, etc.

A wide selection of journals (inclusive of 9 subjects, more than 200 journals)

Providing 24-hour high-quality service

User-friendly online submission system

Fair and swift peer-review system

Efficient typesetting and proofreading procedure

Display of the result of downloads and visits, as well as the number of cited articles

Maximum dissemination of your research work

Submit your manuscript at: http://papersubmission.scirp.org/

Or contact health@scirp.org 